F. Tahmasebi, A. Mahdavi, On the utility of occupants' behavioural diversity information for building performance simulation: an exploratory case study, Energy \& Buildings 176 (2018) 380-389, DOI: 10.1016/j.enbuild.2018.07.042

Free access to the formal publication until 14 October 2018:

https://authors.elsevier.com/a/1XaA31M7zGwV97

\title{
On the Utility of Occupants' Behavioural Diversity Information for Building Performance Simulation: An Exploratory Case Study
}

\author{
Farhang Tahmasebi, Ardeshir Mahdavi \\ Department of Building Physics and Building Ecology, TU Wien, Vienna, Austria
}

\begin{abstract}
The present study aims at investigating the potential advantages of integrating inter-occupant diversity information into occupant behaviour models used in building performance simulation. To this end, the authors model the operation of windows by occupants in a monitored openplan office at aggregate and individual levels. The models use indoor and outdoor temperature as well as the interaction of these variables to estimate the probability of opening and closing windows in the building located in Vienna, Austria. Subsequently, a number of existing and novel metrics serve to compare the predictive performance of the aggregate and individual models. In addition, a calibrated energy model of the office area incorporates the window operation models to evaluate their potential contribution to the reliability of building performance assessments. The results of this exploratory case study suggest that individual window operation models outperform the aggregate model in capturing the peak and variations of window operation across occupants. This resulted in a more reliable thermal comfort assessment in the free-running season. The individual models, however, overestimated peak heating demand, as compared with the benchmark value resulting from the actual window operations in a single year.
\end{abstract}

\section{HIGHLIGHTS}

- Aggregate and individual models of window operation by occupants in an office.

- Interaction of indoor and outdoor temperature included due to very hot summer days.

- Aggregate model did not reproduce variations of window operation across occupants.

- Capturing active occupants by individual models led to a better comfort assessment.

- Coefficient of discrimination showed models' poor ability to recognize actions. 


\section{INTRODUCTION}

A number of empirical and simulation-aided studies have addressed the possible impact of occupants on the buildings' environmental and energy performance $[1,2,3,4]$. The role of occupant behaviour has been also highlighted as one of the contributing factors in cases of mismatch between the predicted and measured energy performance of buildings $[5,6]$. Consequently, the building simulation community has increased its efforts to improve the reliability of simulation tools through more advanced (e.g., probabilistic) representations of occupant behaviour in buildings $[7,8,9]$. However, several studies have demonstrated that the use of existing occupant behaviour models involves considerable uncertainties and does not necessarily lead to a more reliable building performance assessment $[10,11]$. Specifically, it is shown that without proper treatment of the diversity in occupants' behaviour, probabilistic occupancy-related models fail to provide representative ranges of occupant behaviour possibilities as intended $[12,13]$. To address this issue, different approaches for inclusion of diversity in occupant behaviour modelling efforts have been examined: i) Clustering of occupant types [14], ii) Random selection of occupancy-related traits while maintaining the correlations between the traits [12], iii) Use of the errors associated with estimated coefficients of the aggregate data models [15], and iv) Developing models for each occupant together with meta-models defining the distribution of model coefficients $[16,17]$. However, it has been also suggested that for specific cases, such as large open-plan offices [18], a detailed treatment of occupants' diversity may not be beneficial. Consequently, further studies in this area are needed, as the potential benefits of supplying probabilistic occupant behaviour models with inter-occupant diversity information are not conclusively established. In this context, the current contribution revisits the problem through an explorative case study. Specifically, the study explores two essential questions with regard to the use of probabilistic window operation models: To which extent do the predictions of window operation models that do not reflect behavioural diversity differ from actually observed occupants' behaviour? To what degree does the inclusion of occupants' behavioural diversity enhance the building models' potential to provide reliable estimations of key building performance indicators?

\section{METHOD}

\subsection{Overview}

The present study uses long-term monitored data on indoor and outdoor environment and state of windows in an open-plan office area to model the occupants' operation of windows with and without integration of inter-occupant variations in this regard. Subsequently, using the monitored window operation data, a calibrated model of the office area, and a number of 
existing and novel metrics, the study investigates if modelling the inter-occupant diversity in operation of windows contributes to a more reliable building performance analysis.

\subsection{Building and observational data}

The current study focuses on an office area with seven workstations in Vienna, Austria, where each occupant has access to one manually operable casement window (Figure 1). Six of these workstations are in an open-plan area. In this area, the placement of furniture between working places defines separate zones with one window for each occupant. The air, however, can freely flow across the entire open-plan area. The building is not air-conditioned and it only uses a hydronic heating system to actively maintain thermal comfort in the cold season. In this office, the occupants' presence, state of windows and several environmental parameters (including indoor and outdoor air temperature) are monitored on a continuous basis. The study uses the monitored data from a calendar year (referred to as estimation period) to derive the window operation models. A separate set of data obtained from another calendar year (referred to as validation period) is used to evaluate the performance of the models.

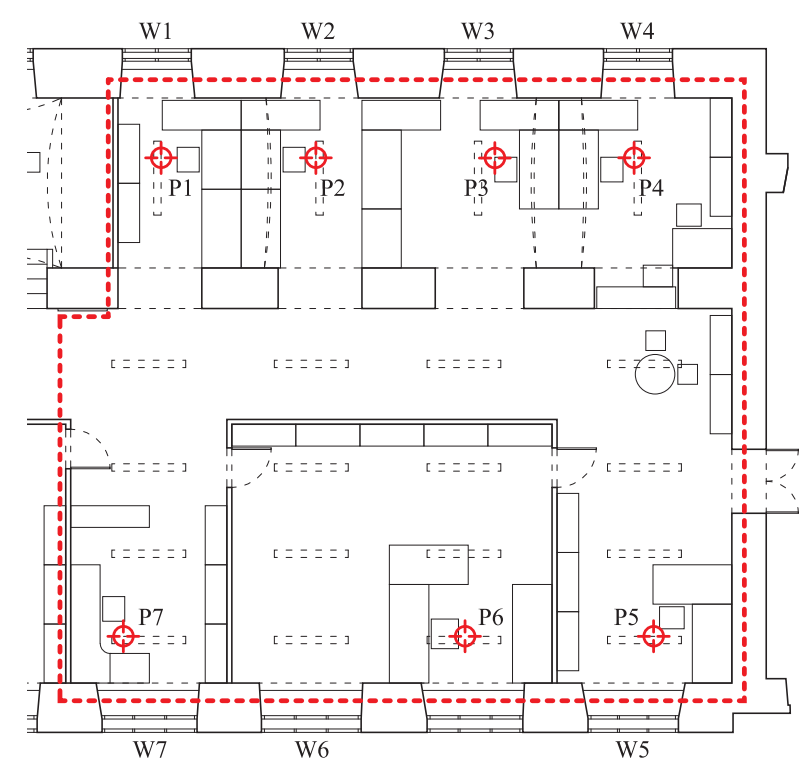

Figure 1: Schematic illustration of the office area, observed occupants and operable windows

\subsection{Office area calibrated simulation model}

The office area was modelled in the building energy simulation tool EnergyPlus 8.8.0. In the zoning scheme, the open-plan south and north-oriented spaces were separated from the central corridor. However, using the network-based multi-zone airflow model of EnergyPlus, the airflows across the external windows and the connected spaces were simulated. The constant input parameters governing airflow simulation in the EnergyPlus model (namely open 
windows discharge coefficient and closed windows air mass flow coefficient) were set based on a previous model calibration effort [19]. Table 1 summarizes the basic information about the calibrated office area energy model.

Table 1: Basic information on the office area and modelling assumptions

\begin{tabular}{|c|c|}
\hline Building data / Modelling assumptions & Value \\
\hline Net conditioned floor area $\left[\mathrm{m}^{2}\right]$ & 187.6 \\
\hline Gross wall area $\left[\mathrm{m}^{2}\right]$ & 120.1 \\
\hline Average window-wall ratio [\%] & 26.7 \\
\hline Exterior walls U-value $\left[\mathrm{W} \cdot \mathrm{m}^{-2} \cdot \mathrm{K}^{-1}\right]$ & 0.65 \\
\hline Exterior windows U-value $\left[\mathrm{W} \cdot \mathrm{m}^{-2} \cdot \mathrm{K}^{-1}\right]$ & 2.79 \\
\hline Exterior windows SHGC [-] & 0.77 \\
\hline Number of occupants [-] & 7 \\
\hline Maximum lighting power density [W.m²] & 4.1 \\
\hline Maximum equipment power density [W. $\left.\mathrm{m}^{-2}\right]$ & 9.9 \\
\hline Number of operable windows [-] & 7 \\
\hline Windows discharge coefficient when open [-] & 0.28 \\
\hline Windows air mass flow coefficient when closed $\left[\mathrm{kg} \cdot \mathrm{s}^{-1} \cdot \mathrm{m}^{-1}\right]$ & $4.15 \times 10^{-4}$ \\
\hline
\end{tabular}

The calibrated building simulation model served as a test bed for evaluation of window operation models with consideration of models' feedback, i.e. the impact of models' output (window states) on models' input (indoor temperature). The building model also made it possible to determine the implications of window operation (and use of different window operation models) for the values of relevant building performance indicators. To this end, the monitored data streams of occupants' presence and use of lights and equipment in the validation year were fed into the model. In addition, the aggregate and individual window operation models (see Section 2.4) were successively integrated into the building model using EnergyPlus runtime language. The study also benefited from a benchmark building model, which contained the actual states of windows based on the monitored data obtained in the validation period. Moreover, the building model was exposed to the outdoor environmental conditions in the validation period using a weather data file generated from on-site weather station measurements. The measured dataset included outdoor air temperature, air humidity, 
atmospheric pressure, global horizontal radiation, diffuse radiation, wind speed, and wind direction. Lastly, due to the stochastic nature of the window operation models, the building model was simulated 50 times to obtain representative ranges of outputs.

It should be also noted that, an ideal unlimited heating system is set in the model to maintain the temperature of different zones according to the measured indoor temperatures in the validation period and to estimate the space heating demands. However, since such a system fully counteracts the impact of window openings, the building model is not strictly applicable to evaluation of window operation models in heating season. Therefore, for the purpose of the current study, the performance of window operation models (in terms of their primary predictions of window states) is only considered in the free-running season (from April 22 to September 25).

\subsection{Window operation models}

To explore the research questions, the authors developed the following two types of window operation models based on the monitored data in the estimation period:

- Aggregate model (AGG), which was derived based on the data obtained from all occupants and windows without any weighting factor;

- Individual models (W1-W7), which were derived based on the data obtained from each occupant and his/her associated window.

Each of the models consists of two logistic regression sub-models for estimation of window opening and closing probabilities. As given in Equations 1 and 2, each logistic regression submodel estimates the probability of opening or closing windows based on indoor and outdoor temperature and their interaction:

$$
\begin{aligned}
& \alpha=\ln \left(\frac{P}{1-P}\right)=\beta_{0}+\beta_{1} \theta_{\text {in }}+\beta_{2} \theta_{\text {out }}+\beta_{3} \theta_{\text {in }} \theta_{\text {out }} \\
& P=\frac{e^{\alpha}}{1+e^{\alpha}}
\end{aligned}
$$

In the equations above, $\alpha$ is the logit function, $\mathrm{P}$ is the probability of opening or closing a window, $\theta_{\text {in }}$ and $\theta_{\text {out }}$ are indoor and outdoor temperature respectively, $\beta_{0}$ is the intercept, and $\beta_{1}$ to $\beta_{3}$ are the regression coefficients. The estimated probabilities of opening and closing actions are subjected to Inverse Transform Sampling Method [20], which involves comparison of the estimated probabilities with a random number between 0 and 1 from the standard uniform distribution, to determine the occurrence of an action. 
Note that, in order to focus the investigation on the inter-occupant diversity representation, the current study adopted a rather simple approach to modelling window operation. Specifically, without conducting a comprehensive variable selection procedure, the models use indoor and outdoor temperatures as explanatory variables, which have been identified by a large number of previous studies as statistically significant variables in explaining the occupants' operation of windows in office buildings $[21,22,23,24,25]$. However, the interaction term was added to the models to capture the effect of indoor temperature depending on outdoor temperature and vice versa. In the previously developed window operation models studied by the authors $[24,25,25,26,27]$, which are derived based on data obtained from buildings located in UK, Switzerland, Denmark, and north-eastern region of the United States, interaction of indoor and outdoor temperature has not been considered. This is, however, especially important in the present study. For in a number of extremely hot summer days in Vienna, natural ventilation cannot serve as a cooling measure and may not be preferable by occupants in the buildings without mechanical cooling.

Moreover, it should be noted that the developed models do no not differentiate between different occupancy phases (such as arrival, intermediate and departure). However, as the occupants in the studied building are requested not to leave the windows open when they leave the office due to storm damage risk, windows are assumed to be closed upon occupants' last departures.

\subsection{Diversity representation}

The present contribution adopts an ideal approach to represent inter-occupant diversity in window operation. Thereby, each individual model is used to predict the behaviour of the occupant, based on whom the model is developed. This strategy was due in part to the small number of occupants in the study. Moreover, it was considered consistent with the study's main aim, namely to explore the potential advantages of integrating diversity in occupant behaviour models.

\subsection{Metrics for models' predictive accuracy}

The following metrics serve to evaluate the window operation models in view of interval-byinterval equivalence of the predicted and monitored window states:

- TPR (True Positive Rate), as the proportion of actual open states, which are correctly predicted.

- FPR (False Positive Rate), denoting the proportion of actual closed states, which are wrongly predicted. 
- TNR (True Negative Rate), denoting the proportion of actual closed states, which are correctly predicted.

- FNR (False Negative Rate) as the proportion of actual open states, which are wrongly predicted.

In addition, three indicators examine the dynamics and overall aspects of the predicted window operations as follows:

- Overall fraction of open state, as the total window opening time divided by the observation time.

- Mean number of actions per day averaged over the observation time.

- Median open state duration, to capture the durations for which windows are left open.

The aforementioned indicators have been widely used in previous studies pertaining to the evaluation of occupant behaviour models $[25,28]$. However, in order to measure the models' ability to discriminate between occurrence and nonoccurrence of window openings and closings, the authors deployed a rather novel metric in this field, namely the coefficient of discrimination. This metric is suggested by Tjur [29] in the context of logistic regression as an analogue to the coefficient of determination (R-squared) in ordinary regression models. The coefficient of discrimination $(\mathrm{CoD})$ is calculated as follows:

$$
\operatorname{CoD}=\overline{\hat{\pi}}_{1}-\overline{\hat{\pi}}_{0}
$$

Where $\overline{\hat{\pi}}_{1}$ and $\overline{\hat{\pi}}_{0}$ denote the average of fitted values (estimated probabilities of window opening or closing), respectively, for the so-called successes (intervals, in which an opening or a closing action has been observed) and the failures (intervals, in which an opening or a closing action has not been observed). Similar to R-squared in linear regression, coefficient of discrimination takes values between 0 and 1. A value of 0 corresponds to "no explanatory power" (all fitted values are equal), and a value of 1 corresponds to "perfect fit" (the fitted values coincide with the observations) [29]. This indicator offers two advantages in evaluation of occupant behaviour models: Firstly, it is obtained without conducting Monte Carlo simulation of the model (as it deploys the estimated probabilities and not the randomly-sampled states). Secondly, it does not require the inclusion of the models' feedback as it examines the models' predictions in a set of disconnected time intervals. 


\subsection{Building performance indicators}

To capture the office area performance in heating and free-running seasons, the building energy model estimates the following indicators:

- Annual heating demand per floor area $\left[\mathrm{kWh} \cdot \mathrm{m}^{-2}\right]$, which gives the total heating energy required in a year to maintain the occupants' desired temperature set-points.

- Peak heating demand per floor area $\left[\mathrm{W} . \mathrm{m}^{-2}\right]$, which gives the maximum heating energy required in a 15-min interval to maintain temperature set-points.

- Time outside EN15251 comfort zone [\%], which denotes the fraction of occupied time in the free-running season, in which operative temperature is outside EN15251 Category III limits.

\section{RESULTS}

\subsection{Window operation models}

Table 2 provides the estimated coefficients for the individual and aggregate window opening and closing models. Figure 2 illustrates slices through the response surface of individual and aggregate models at an outdoor temperature of $20^{\circ} \mathrm{C}$. Figure 3 depicts such slices at indoor temperatures of $20^{\circ} \mathrm{C}$ and $30^{\circ} \mathrm{C}$.

In addition, to better illustrate the usefulness of the interaction term in capturing the occupants' interaction with windows in very hot summer days, Figure 4 shows, as example, the surface plot of the individual window opening model W5 in comparison with a model fitted without the interaction term. Besides, to numerically test if the inclusion of interaction term has enhanced the quality of the models, Table 3 compares the AIC values of the derived window opening and closing models with those of models without interaction term. Note that, AIC is a measure of the relative quality of statistical models, which aims at finding a trade-off between the goodness of fit of and simplicity of the models by favouring a high likelihood function value and penalizing the number of parameters. When comparing candidate models for a specific dataset, the preferred model is the one with the lowest AIC value. 
Table 2: Estimated coefficients for logistic regression models of window opening and closing based on the individual windows (W1 - W7) and aggregate data.

\begin{tabular}{|c|c|c|c|}
\hline $\begin{array}{l}\text { Window } \\
\text { operation model }\end{array}$ & $\begin{array}{l}\text { Variables and } \\
\text { terms }\end{array}$ & $\begin{array}{l}\text { Opening } \\
\text { coefficients }\end{array}$ & $\begin{array}{c}\text { Closing } \\
\text { coefficients }\end{array}$ \\
\hline \multirow{4}{*}{$\begin{array}{l}\text { Individual } \\
\text { W1 }\end{array}$} & Intercept & -10.4233 & 16.6416 \\
\hline & $\theta_{\text {in }}$ & 0.0905 & -0.7013 \\
\hline & $\theta_{\text {out }}$ & 0.2047 & -0.5011 \\
\hline & Interaction & -0.0034 & 0.0186 \\
\hline \multirow{4}{*}{$\begin{array}{l}\text { Individual } \\
\text { W2 }\end{array}$} & Intercept & -12.2998 & 27.2410 \\
\hline & $\theta_{\text {in }}$ & 0.3135 & -1.1479 \\
\hline & $\theta_{\text {out }}$ & 0.1075 & -1.1022 \\
\hline & Interaction & -0.0032 & 0.0424 \\
\hline \multirow{4}{*}{$\begin{array}{l}\text { Individual } \\
\text { W3 }\end{array}$} & Intercept & -28.3577 & 7.9830 \\
\hline & $\theta_{\text {in }}$ & 0.9783 & -0.4323 \\
\hline & $\theta_{\text {out }}$ & 0.9343 & -0.3756 \\
\hline & Interaction & -0.0363 & 0.0144 \\
\hline \multirow{4}{*}{$\begin{array}{l}\text { Individual } \\
\text { W4 }\end{array}$} & Intercept & -31.2056 & 29.7625 \\
\hline & $\theta_{\text {in }}$ & 1.0775 & -1.3678 \\
\hline & $\theta_{\text {out }}$ & 0.4713 & -1.0668 \\
\hline & Interaction & -0.0195 & 0.0454 \\
\hline \multirow{4}{*}{$\begin{array}{l}\text { Individual } \\
\text { W5 }\end{array}$} & Intercept & -49.5078 & 15.5018 \\
\hline & $\theta_{\text {in }}$ & 1.9324 & -0.7982 \\
\hline & $\theta_{\text {out }}$ & 1.5071 & -0.6517 \\
\hline & Interaction & -0.0617 & 0.0283 \\
\hline \multirow{4}{*}{$\begin{array}{l}\text { Individual } \\
\text { W6 }\end{array}$} & Intercept & -22.4190 & 23.7542 \\
\hline & $\theta_{\text {in }}$ & 0.8031 & -1.0475 \\
\hline & $\theta_{\text {out }}$ & 0.3130 & -0.8246 \\
\hline & Interaction & -0.0123 & 0.0332 \\
\hline \multirow{4}{*}{$\begin{array}{l}\text { Individual } \\
\text { W7 }\end{array}$} & Intercept & -13.7355 & 25.3944 \\
\hline & $\theta_{\text {in }}$ & 0.4225 & -1.1495 \\
\hline & $\theta_{\text {out }}$ & 0.0343 & -0.9280 \\
\hline & Interaction & -0.0007 & 0.0374 \\
\hline \multirow{4}{*}{$\begin{array}{l}\text { Aggregate } \\
\text { (AGG) }\end{array}$} & Intercept & -10.6882 & 23.9665 \\
\hline & $\theta_{\text {in }}$ & 0.2187 & -1.0969 \\
\hline & $\theta_{\text {out }}$ & 0.2100 & -0.9172 \\
\hline & Interaction & -0.0052 & 0.0376 \\
\hline
\end{tabular}



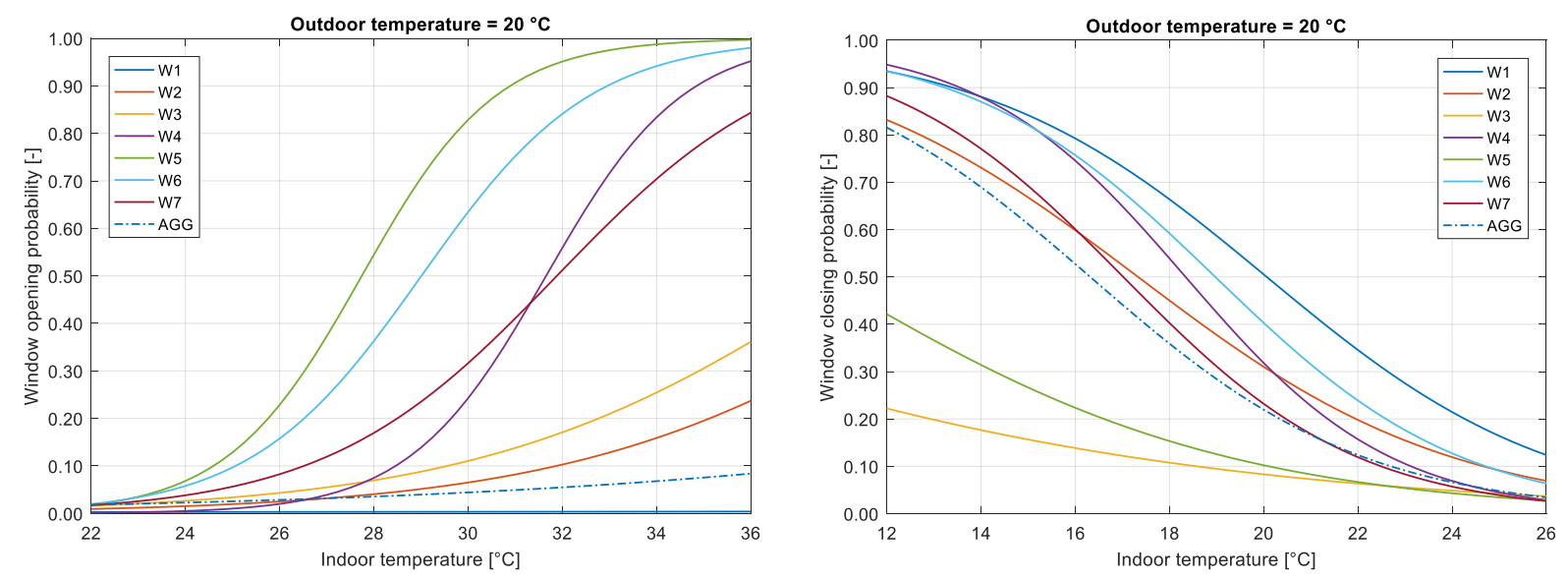

Figure 2: Slices through the response surface of individual and aggregate models for opening (left) and closing (right) of windows at an outdoor temperature of $20^{\circ} \mathrm{C}$.
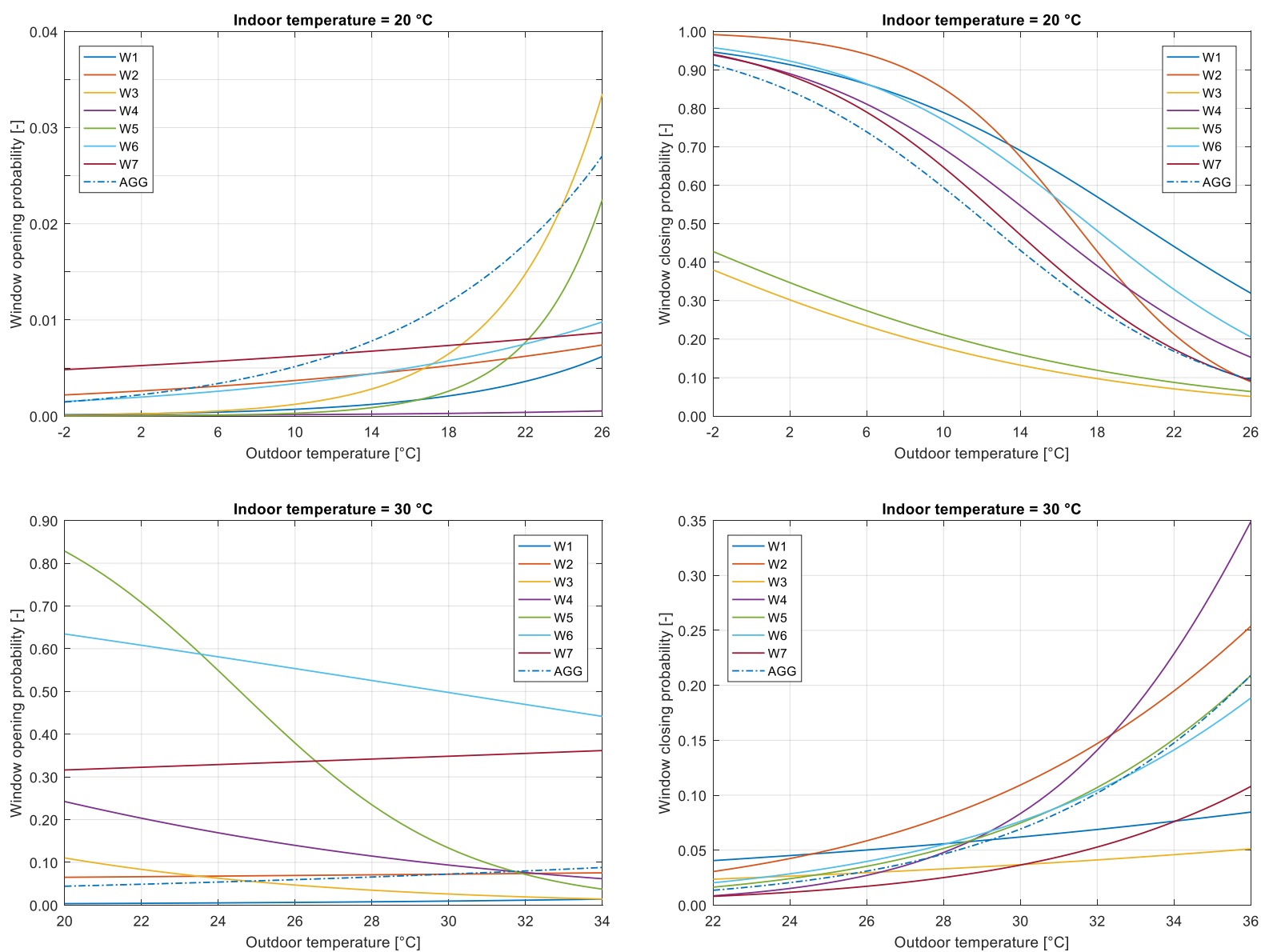

Figure 3: Slices through the response surface of individual and aggregate models for opening (left) and closing (right) of windows at indoor temperatures of $20^{\circ} \mathrm{C}$ (top) and $30^{\circ} \mathrm{C}$ (bottom). 

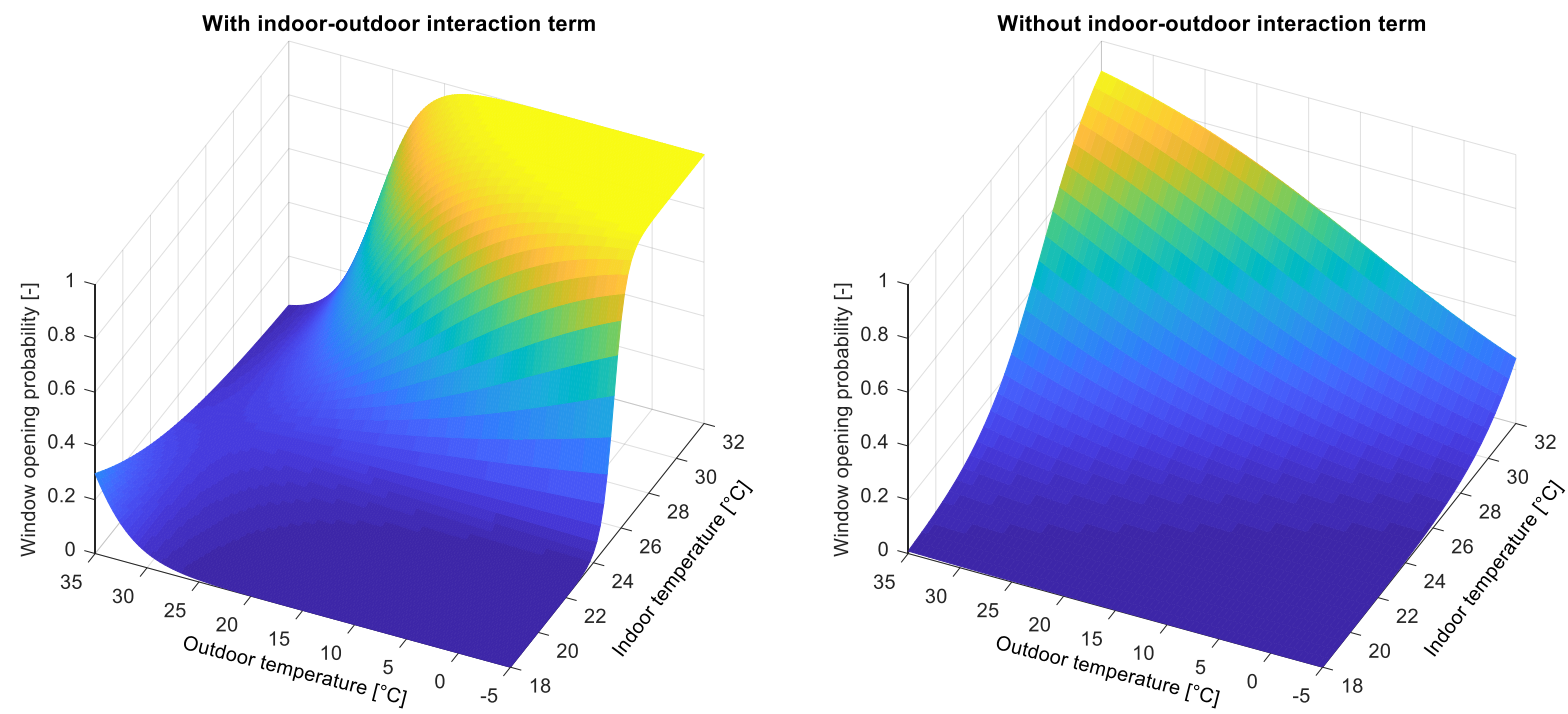

Figure 4: Three-dimensional surface plot of the individual window operation model W5 with indoor-outdoor interaction term (left) in comparison with the fitted model without interaction term (right).

Table 3: Obtained AIC values for window opening and closing models with and without including interaction of indoor and outdoor temperature

\begin{tabular}{ccccc}
\hline \multirow{2}{*}{ Window } & \multicolumn{2}{c}{ With interaction term } & \multicolumn{2}{c}{ Without interaction term } \\
\cline { 2 - 5 } & Opening & Closing & Opening & Closing \\
\hline W1 & 225.7 & 88.3 & 223.8 & 86.5 \\
W2 & 1061.1 & 463.5 & 1059.2 & 471.8 \\
W3 & 1314.4 & 1057.7 & 1329.9 & 1058.2 \\
W4 & 475.5 & 269.7 & 476.1 & 272.7 \\
W5 & 596.4 & 500.1 & 617.7 & 500.4 \\
W6 & 1260.7 & 721.5 & 1261.2 & 733.1 \\
W7 & 1078.0 & 636.2 & 1076.0 & 644.6 \\
AGG & 6468.9 & 3870.7 & 6469.6 & 3948.1 \\
\hline
\end{tabular}

\subsection{Occupants' interactions with windows}

Table 4 provides the metrics obtained from interval-by-interval comparison of predicted and monitored window states in the entire office area throughout the validation period. In addition, the models' ability to discriminate window opening and closing actions is captured with values 
of the coefficient of discrimination given in Table 5. This table also includes the coefficient of discrimination obtained from two widely-used existing window operation models developed by Rijal et al [23] and Haldi and Robinson [25] for reference. The first reference model [23] estimates the window opening and closing probabilities based on outdoor and operative temperature, when operative temperature is below or above a comfort temperature dead-band. The second reference model [25] estimates the probability of opening and closing actions at arrival, intermediate and departure intervals based on a number of occupancy-related and environmental independent variables.

Moreover, to better compare the performance of the models in discriminating window opening actions, Figure 5 shows the predicted window opening probabilities by aggregate and individual models for separate sets of intervals as follows: a) intervals with a closed window, where an opening is observed (i.e. the ideal prediction equals 1 ) in the top histograms; b) intervals with a closed window, where an opening is not observed (i.e. the ideal prediction is $0)$ in the bottom histograms.

Further aspects of window operation predictions, namely, overall fraction of open state, number of opening actions, and open state durations are summarized in Table 6 . It provides the observed and predicted values of the metrics for individual occupants/windows in the freerunning season, together with the mean and standard deviation of the metrics across occupants. To facilitate the comparison of models' performance in capturing the variations in occupants' interactions with windows, Figure 6 shows the coefficient of variation of the metrics (as the ratio of standard deviation to the mean) obtained from the observations and the predictions made by the aggregate and individual models. Note that the values of the metrics in Table 4 and Table 6 are obtained from averaging the models' predictions in 50 Monte Carlo runs.

Table 4: Values of TPR, FPR, TNR, FNR for aggregate and individual window operation models
\begin{tabular}{ccccc} 
in the free-running validation period \\
Model(s) & $\begin{array}{r}\text { TPR } \\
{[\%]}\end{array}$ & $\begin{array}{c}\text { FPR } \\
{[\%]}\end{array}$ & $\begin{array}{c}\text { TNR } \\
{[\%]}\end{array}$ & $\begin{array}{c}\text { FNR } \\
{[\%]}\end{array}$ \\
\hline Aggregate & 29.2 & 4.3 & 95.7 & 70.8 \\
Individuals & 47.1 & 5.9 & 94.1 & 52.9 \\
\hline
\end{tabular}


Table 5: Values of Coefficient of Discrimination (CoD) for window opening and closing actions obtained from aggregate and individual window operation models in the validation period

\begin{tabular}{ccc}
\hline Model(s) & CoD $_{\text {opening }}[-]$ & CoD $_{\text {closing }}[-]$ \\
\hline Aggregate & 0.008 & 0.082 \\
Individuals & 0.034 & 0.127 \\
Reference 1 [23] & 0.044 & 0.008 \\
Reference 2 [25] & 0.013 & 0.012 \\
\hline
\end{tabular}
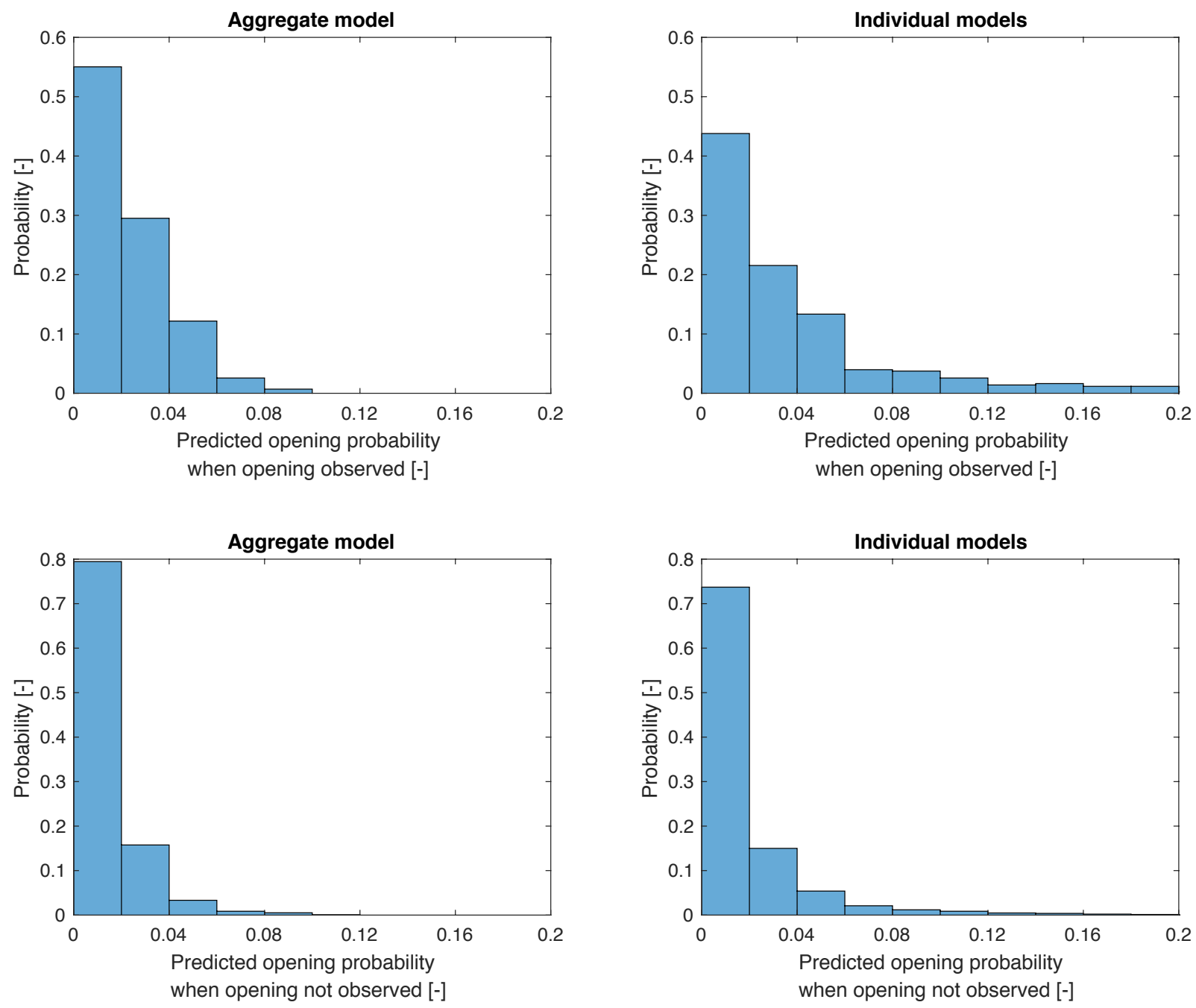

Figure 5: Histograms of predicted window opening probabilities by aggregate (left) and individual (right) models for the intervals where the closed windows were opened (top) and for the intervals where the windows remained closed (bottom). 
Table 6: Observed and predicted mean values of fraction of open state, openings per day, and median open state duration for individual windows along with inter-occupant mean and standard deviation of the metrics

\begin{tabular}{|c|c|c|c|c|c|c|c|c|c|}
\hline \multirow{2}{*}{ Window } & \multicolumn{3}{|c|}{ Overall fraction of open state [\%] } & \multicolumn{3}{|c|}{ Opening actions per day $\left[\mathrm{d}^{-1}\right]$} & \multicolumn{3}{|c|}{ Median open state duration [h] } \\
\hline & Observed & $\begin{array}{c}\text { Aggregate } \\
\text { model }\end{array}$ & $\begin{array}{c}\text { Individual } \\
\text { models }\end{array}$ & Observed & $\begin{array}{c}\text { Aggregate } \\
\text { model }\end{array}$ & $\begin{array}{l}\text { Individual } \\
\text { models }\end{array}$ & Observed & $\begin{array}{c}\text { Aggregate } \\
\text { model }\end{array}$ & $\begin{array}{c}\text { Individual } \\
\text { models }\end{array}$ \\
\hline W1 & 2.5 & 5.5 & 0.5 & 0.18 & 0.45 & 0.07 & 2.00 & 2.38 & 1.46 \\
\hline W2 & 7.8 & 6.5 & 4.8 & 0.46 & 0.48 & 0.50 & 3.75 & 2.57 & 1.62 \\
\hline W3 & 3.6 & 7.5 & 8.8 & 0.18 & 0.52 & 0.55 & 4.00 & 2.68 & 3.13 \\
\hline W4 & 10.8 & 6.8 & 7.3 & 0.48 & 0.54 & 0.52 & 4.75 & 2.31 & 2.56 \\
\hline W5 & 8.8 & 7.2 & 19.7 & 0.50 & 0.56 & 1.02 & 3.25 & 2.43 & 3.43 \\
\hline W6 & 9.3 & 5.0 & 11.7 & 0.50 & 0.43 & 1.04 & 2.75 & 1.99 & 1.52 \\
\hline W7 & 17.8 & 6.7 & 13.6 & 0.69 & 0.52 & 0.83 & 4.13 & 2.50 & 3.37 \\
\hline Mean & 8.7 & 6.5 & 9.5 & 0.43 & 0.50 & 0.65 & 3.52 & 2.41 & 2.44 \\
\hline SD & 4.7 & 0.9 & 5.8 & 0.17 & 0.04 & 0.32 & 0.86 & 0.21 & 0.83 \\
\hline
\end{tabular}

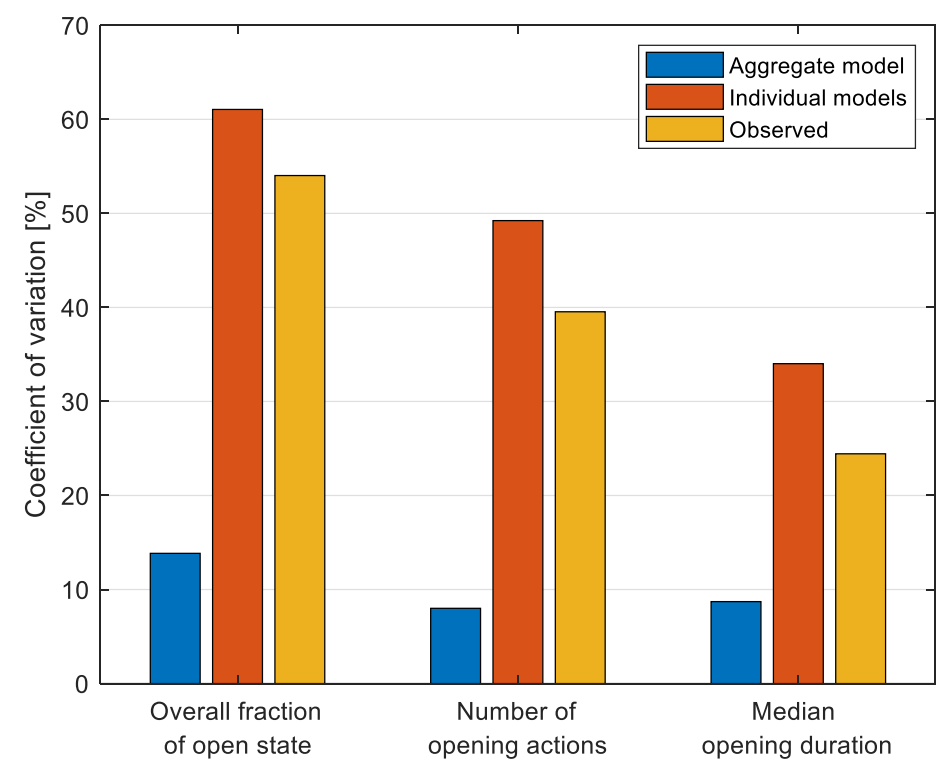

Figure 6: Coefficient of variation of the overall fraction of open state, number of opening actions, and median opening duration across the occupants obtained from observations, aggregate and individual window operation models

\subsection{Building performance indicators}

Table 7 gives the obtained values of building performance indicators, namely, annual and peak heating demands, and the percentage of occupied time outside EN15251 comfort limits. It includes the outputs of the benchmark model (with the monitored window operation data), along with the mean and standard deviation of the predictions provided by the building models equipped with the aggregate and individual window operation models. To put the models' 
performance in context, Table 7 also provides, for comparison purposes, the results obtained from two simple window operation models. The first one is a non-probabilistic model, which assumes windows are opened if indoor temperature is above $26^{\circ} \mathrm{C}$. The second one assumes windows are always closed. Note that the latter model is presented for reference only and is not a realistic alternative.

To better interpret the obtained results, Figure 7 illustrates the relative error of the estimated building performance indicators as compared to the benchmark model. Note that, due to the very large overestimation of discomfort by the model with closed windows, the output of this model is not included in Figure 7.

Table 7: Building performance indicators obtained from the building models equipped with monitored window operation (benchmark), aggregate and individual window operation models, and two simple non-probabilistic models

\begin{tabular}{cccc}
\hline $\begin{array}{c}\text { Window } \\
\text { operation model(s) }\end{array}$ & $\begin{array}{c}\text { Annual heating } \\
\text { demand [kWh.m }{ }^{-2} \text { ] }\end{array}$ & $\begin{array}{c}\text { Peak heating } \\
\text { demand [W. } \mathbf{m}^{-2} \text { ] }\end{array}$ & $\begin{array}{c}\text { Time outside EN15251 } \\
\text { comfort zone [\%] }\end{array}$ \\
\hline Benchmark & 64.7 & 89.3 & 5.6 \\
Aggregate & $67.4 \pm 0.5$ & $95.5 \pm 15.5$ & $8.8 \pm 0.5$ \\
Individuals & $68.0 \pm 0.5$ & $111.0 \pm 20.8$ & $4.6 \pm 0.3$ \\
Open if $\theta_{\text {in }}>26$ & 62.8 & 60.4 & 2.8 \\
Closed & 62.4 & 45.5 & 25.0 \\
\hline
\end{tabular}

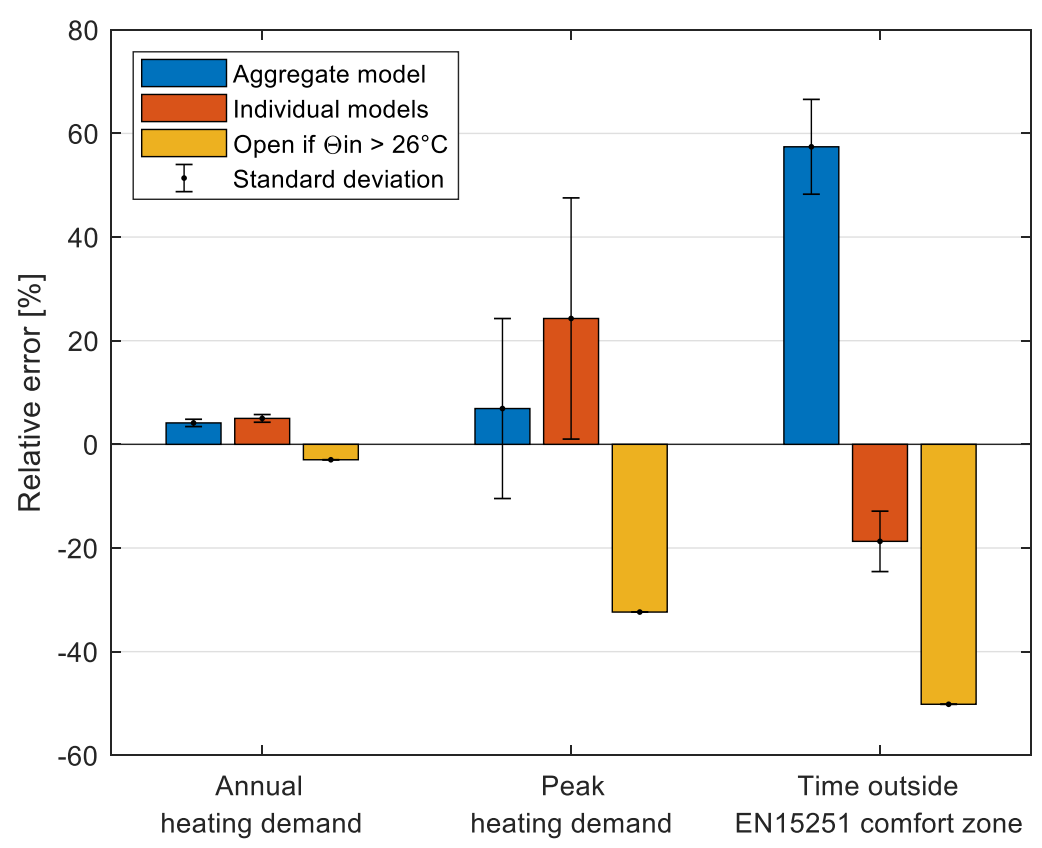

Figure 7: Relative error of the performance indicators obtained from the building model with aggregate and individual window operation models as well as a simple non-probabilistic model 


\section{DISCUSSION}

\subsection{Window operation models}

As it can be seen in Figure 2 and Figure 3, the observed occupants in the office area show a noticeable spread in view of the probabilities of opening and closing windows in different indoor and outdoor temperatures. Moreover, inclusion of the interaction term has benefited the models toward capturing the declining interest of occupants in natural ventilation in very hot summer days. As it can be seen in the top left plot in Figure 3, with an indoor temperature of $20^{\circ} \mathrm{C}$ and outdoor temperatures such as -2 to $26^{\circ} \mathrm{C}$, the probability of opening window increases with the rise of outdoor temperature. However, in extreme summer conditions (for example with an indoor temperature of $30^{\circ} \mathrm{C}$ ), for a number of occupants, the probability of opening the window is decreasing when the outdoor temperature rises (Figure 3, bottom left plot). A similar pattern can be seen even more uniformly in terms of estimated window closing probabilities (Figure 3, right hand side plots). The large impact of the indoor-outdoor interaction term in transforming the response surface of the window opening model for one of the occupants (W5) can be seen in Figure 4 as well.

The contribution of the interaction term in enhancing the quality of the models has been also shown in Table 3, as 12 out of 16 developed logistic regression models display lower AIC values as compared with models which do not include interaction of indoor and outdoor temperature.

\subsection{Occupants' interactions with windows}

The results provided in Table 4 indicate that individual window operation models outperform the aggregate model in correctly predicting open states (TPR of $47.1 \%$ versus $29.2 \%$ ), whereas their false predictions of open states are only slightly more frequent than those of the aggregate model (FPR of $5.9 \%$ versus $4.3 \%$ ). The individual models also generate fewer false predictions of closed states (FNR of $52.9 \%$ versus $70.8 \%$ ) and their performance in correctly predicting the closed states is very close to that of the aggregate model.

The low values of the coefficient of discrimination given in Table 5 for both aggregate and individual models suggest that their performance in this regard is far from satisfactory. This can be also inferred from the considerable overlap in the upper and lower histograms in Figure 5. Note that a high discrimination potential would have resulted in concentration of the upper histogram mass close to the right endpoint and the lower histogram mass close to the left endpoint. However, the similar values obtained from the predictions by two reference models reveal that this poor performance is not exclusively attributable to the models developed in this study. In fact, this may be rather explained by the inherent limit to the extent to which one can predict the exact time of occupants' control-oriented actions. Nonetheless, the higher values 
of the coefficient of discrimination for the individual models, as compared with the aggregate model, suggests that the integration of diversity into the models has to some extent improved their ability in discriminating window opening and closing actions. This can be also seen in Figure 5, as the individual models have, to some extent, pushed the upper histogram mass toward the right endpoint (which has exceeded their negative impact in the lower histogram).

Moreover, from the metrics provided for individual windows in Table 6, an interesting observation can be made concerning the overall fraction of open state: Even though each occupant's model is based on the data obtained from the same occupant in the estimation period, the individual models have not identified the occupant who has kept the window open the longest. Based on observations, W7 shows the maximum overall fraction of open state with $17.8 \%$. However, in case of the individual models' predictions, the maximum overall fraction of open state belongs to W5 with $19.7 \%$. Nonetheless, and arguably more importantly, the individual models have outperformed the aggregate model in capturing the peak operation of individual windows. In fact, the aggregate model's lack of sensitivity to inter-occupant diversity leads to the prediction that no occupant keeps the windows open longer than $7.5 \%$ of the time in free-running season. This has implications for the assessment of thermal comfort, which will be discussed in the next section.

As it can be seen from the mean values provided in Table 6, individual models do not show a better performance in predicting the average tendencies in terms of number of opening actions and opening duration. In fact, they even show a larger error in the average number of actions across occupants. However, the combination of an overestimation of the number of openings and an underestimation of the opening duration has resulted in a better estimation of the overall fraction of open state. The aggregate model, however, has largely underestimated the mean duration of openings and slightly overestimated the number of actions in the office. This has resulted in an underestimation of the overall fraction of open state. As for capturing the variations in occupants' interactions with windows, Figure 6 shows that the aggregate model has not captured the diversity among occupants. The individual models, however, have reproduced the variation of the metrics values across occupants, albeit with a slight overestimation.

\subsection{Building simulation results}

From Table 7, it can be seen that the aggregate and individual window operation models provide close estimations of the annual heating demand. However, the small standard deviation of the estimated values may suggest that use of probabilistic window operation models for estimation of annual heating demand is not particularly productive. The nonprobabilistic models, which disregard the possibility of window opening in heating season, 
provide reasonable estimates of annual heating demand. However, the non-probabilistic models do not capture the peak heating demands resulting from occasional operation of windows in winter. In contrast, the aggregate and individual probabilistic models overestimate the peak values, which is more noticeable in case of individual models. Nonetheless, the benchmark peak heating demand (as one instance of possible annual peak demands in the building operation phase) is within one standard deviation of the mean predicted value by the aggregate model and is very close to that of the individual models (Figure 7).

With regard to the estimation of thermal comfort in the free-running season (see Figure 7), the individual window operation models outperformed the aggregate probabilistic model and the non-probabilistic ones. As mentioned before, even though the individual models could not identify the most active window-operating occupant in the validation period, they have done well in capturing the peak and the variations in the fraction of open state among occupants (see Table 6 and Figure 6). This has served the building model with individual window operation models to better estimate the occupants' thermal comfort in the free-running season (see Table 7 and Figure 7).

Delving further into this finding, one can hypothesize that, in a free-running open-plan office area with multiple occupants and windows, when few occupants open the windows (and, for example, enable cross ventilation) the rest of the occupants may not feel the need to open the windows anymore (perhaps, amongst other things, to avoid draught problem). Therefore, firstly, the occupants who open the windows more frequently may be different from time to time (as is the case in the estimation and validation periods in the present study). Secondly, due to the smaller number of active occupants, as compared with the passive ones (perhaps because in an open-plan office, opening a small number of windows may suffice to change the indoor conditions), such adaptive actions may not be captured in window operation models relying on the aggregate observations.

In this case study, the aggregate probabilistic window operation model did not include the influence of active occupants and therefore overestimated thermal discomfort. In contrast, the non-probabilistic model, which opens all the windows when indoor temperature exceeds $26^{\circ} \mathrm{C}$, underestimated the thermal discomfort due to an overestimation of air change rate (which may not be even preferable for occupants). The individual probabilistic models, however, could reproduce window operations by active occupants in a more realistic manner, which led to a better thermal comfort assessment in the free-running season.

\section{CONCLUSION}

The study presented in this paper was limited to seven occupants in a specific office space in Vienna, Austria. The window operation models were developed and tested based on the data 
obtained from the same building. The study also examined an ideal case in representation of inter-occupant diversity, as the models estimated for individual occupants were used to predict the same occupants' interactions with windows. Moreover, the window operation models did not address the interacting dynamics of occupant behaviour in an open-plan office (for example, by considering the state of other windows in the office area). Consequently, the authors do not suggest that the results of the study can be simply generalized. Rather, the main purpose of the present exploratory case study was to outline a methodological path to be further pursued in a series of future studies that will hopefully benefit from growing efforts in global occupancy-related data collection and sharing. Nonetheless, the results suggest that integrating inter-occupant diversity in window operation models may be beneficial in terms of:

- Discrimination between open and closed states of the windows;

- Discrimination between occurrence and nonoccurrence of window opening and closing actions;

- Estimation of the peak and variations of window operation across occupants, which cannot be captured by models derived from aggregate data;

- Assessing thermal comfort in an open-plan space, in which each occupant's adaptive actions could change the indoor environmental conditions of the whole space and thus influence other occupants.

With regard to the estimation of annual heating demand, the study did not reveal a benefit in using the aggregate or individual probabilistic models. As for the assessment of peak heating demand, the aggregate model showed a reasonable performance. The integration of interoccupant diversity led to an overestimation of peak heating demand, as compared with the benchmark value resulting from the actual window operations in a single year.

Note that, while the present study may point to the importance of inter-occupant diversity information in window operation models for specific building performance queries, it does not offer a generic and practical approach for the pervasive provision and systematic inclusion of such information in routine building performance simulation processes. Specifically, to provide effective simulation-based design support, occupant models would have to be based on statistically representative diversity information. As such information is currently unavailable, care should be taken in extrapolating from the results obtained from the few available empirical sources of data - such as what this study was based on. This highlights the need for larger numbers of coordinated behavioural studies including diversity considerations. Moreover, concerted efforts are necessary to formulate and implement practically feasible inclusion of diversity information in models of occupants' control-oriented actions in buildings. 


\section{ACKNOWLEDGEMENTS}

The authors gratefully acknowledge that the research presented in this paper largely benefited from the authors' participation in IEA-EBC Annex 66 (Definition and Simulation of Occupant Behavior in Buildings).

\section{REFERENCES}

[1] Gill, Z.M., Tierney, M.J., Pegg, I.M., and N. Allan. 2010. Low- energy dwellings: the contribution of behaviors to actual performance, Building Research \& Information 38:5, 491-508. https://doi.org/10.1080/09613218.2010.505371.

[2] T. Hong, H.W. Lin, Occupant Behavior: Impact on Energy Use of Private Offices, ASim 2012.

[3] Samuelson, H.W., Ghorayshi, A., and C.F. Reinhart. 2016. Analysis of a simplified calibration procedure for 18 design-phase building energy models, Journal of Building Performance Simulation 9 (2016), https://doi.org/10.1080/19401493.2014.988752.

[4] A. Mahdavi, The Human Dimension of Building Performance Simulation, Proceedings of the 12th Conference of The International Building Performance Simulation Association, 2011, K16 - K33.

[5] P. de Wilde, The gap between predicted and measured energy performance of buildings: A framework for investigation, Automation in Construction, Volume 41, May 2014, Pages 40-49, https://doi.org/10.1016/j.autcon.2014.02.009.

[6] T. Hong, S.C. Taylor-Lange, S. D'Oca, D. Yan, S.P. Corgnati, Advances in research and applications of energy-related occupant behaviour in buildings, Energy and Buildings, Volume 116, 15 March 2016, Pages 694-702, https://doi.org/10.1016/j.enbuild.2015.11.052.

[7] D. Yan, T. Hong, B. Dong, A. Mahdavi, S. D'Oca, I. Gaetani, X. Feng, IEA EBC Annex 66: Definition and simulation of occupant behavior in buildings, Energy and Buildings, Volume 156, 1 December 2017, Pages 258-270, https://doi.org/10.1016/j.enbuild.2017.09.084.

[8] D. Yan, W. O'Brien, T. Hong, X. Feng, H.B. Gunay, F. Tahmasebi, A. Mahdavi, Occupant behaviour modelling for building performance simulation: Current state and future challenges, Energy and Buildings, 107, 2015, pp. 264-278, https://doi.org/10.1016/j.enbuild.2015.08.032.

[9] Schweiker, M. 2017. Understanding occupants' behaviour for energy efficiency in buildings, Current Sustainable Renewable Energy Reports (2017) 4:8-14, https://doi.org/10.1007/s40518017-0065-5.

[10] Mahdavi, A., 2015. Common fallacies in representation of occupants in building performance simulation, Proceedings of the 2nd IBPSA-Italy Conference, 1-7, ISBN: 978-88-6046-074-5.

[11] Tahmasebi, F., Mahdavi, A. 2016. An inquiry into the reliability of window operation models in building performance simulation, Building and Environment 105 (2016), 343-357, https://doi.org/10.1016/j.buildenv.2016.06.013.

[12] O'Brien, W., H.B. Gunay, F. Tahmasebi, and A. Mahdavi. 2017. A preliminary study of representing the inter-occupant diversity in occupant modelling, Journal of Building Performance Simulation, 10:5-6, 509-526, https://doi.org/10.1080/19401493.2016.1261943.

[13] Tahmasebi, F., Mahdavi, A. 2017. The sensitivity of building performance simulation results to the choice of occupants' presence models: a case study. Journal of Building Performance Simulation, 10:5-6, 625-635, https://doi.org/10.1080/19401493.2015.1117528.

[14] Reinhart, C.F. 2004. Lightswitch-2002: a model for manual and automated control of electric lighting and blinds, Solar Energy 77(1) 15-28, https://doi.org/10.1016/j.solener.2004.04.003.

[15] Gunay HB, O'Brien W, Beausoleil-Morrison I, 2015. Implementation and comparison of existing occupant behaviour models in EnergyPlus, Journal of Building Performance Simulation (2015), https://doi.org/10.1080/19401493.2015.1102969. 
[16] Haldi F, 2013. A probabilistic model to predict building occupants' diversity towards their interactions with the building envelope, Proceedings of BS2013, 1475-1482.

[17] Haldi, F., Calì, D., Andersen, R.K., Wesseling, M., and D. Müller. 2017. Modelling diversity in building occupant behaviour: a novel statistical approach, Journal of Building Performance Simulation, 10:5-6, 527-544, https://doi.org/10.1080/19401493.2016.1269245.

[18] Gilani, S., O'Brien, W., Gunay, B. 2018. Simulating occupants' impact on building energy performance at different spatial scales, Building and Environment 132, 327-337, https://doi.org/10.1016/j.buildenv.2018.01.040.

[19] Tahmasebi, F., Mahdavi, A. 2012. Optimization-based simulation model calibration using sensitivity analysis, 7th Conference of IBPSA-CZ, Brno, Czech Republic.

[20] E. Zio, The Monte Carlo Simulation Method for System Reliability and Risk Analysis, first ed., Springer, London, 2013.

[21] V. Fabi, R.V. Andersen, S. Corgnati, B.W. Olesen, Occupants' window opening behaviour: A literature review of factors influencing occupant behaviour and models, Building and Environment, Volume 58, December 2012, Pages 188-198, https://doi.org/10.1016/j.buildenv.2012.07.009.

[22] S. Pan, Y. Xiong, Y. Han, X. Zhang, L. Xia, S. Wei, J. Wu, M. Han, A study on influential factors of occupant window-opening behavior in an office building in China, Building and Environment, Volume 133, April 2018, Pages 41-50, https://doi.org/10.1016/j.buildenv.2018.02.008.

[23] Rijal H.B, Tuohy P., Humphreys M.A., Nicol J.F., Samuel A., Clarke J., 2007. Using results from field surveys to predict the effect of open windows on thermal comfort and energy use in buildings. Energy and Buildings, 39 (2007), pp 823-836, doi:10.1016/j.enbuild.2007.02.003.

[24] Yun G.U., Steemers K., 2008. Time-dependent occupant behaviour models of window control in summer. Building and Environment, 43(2008), pp 1471-1482, doi:10.1016/j.buildenv.2007.08.001.

[25] Haldi F., Robinson D., 2009. Interactions with window openings by office occupants. Building and Environment, 44(2009), pp 2378-2395, doi:10.1016/j.buildenv.2009.03.025.

[26] Andersen, R., Fabi, V., Toftum, J., Corgnati, S.P., and BW Olesen. 2013. Window opening behaviour modelled from measurements in Danish dwellings, Building and Environment 69, 101-113, https://doi.org/10.1016/j.buildenv.2013.07.005.

[27] J.G.C. Laurent, H.W. Samuelson, Y. Chen, The impact of window opening and other occupant behavior on simulated energy performance in residence halls, Building Simulation 10 (6), 963976, https://doi.org/10.1007/s12273-017-0399-3.

[28] Schweiker M., Haldi F., Shukuya M., Robinson D., 2012. Verification of stochastic models of window opening behavior for residential buildings, Journal of Building Performance Simulation, 5(1), 55-74, https://doi.org/10.1080/19401493.2011.567422.

[29] Tue Tjur, 2009. Coefficients of Determination in Logistic Regression Models - A New Proposal: The Coefficient of Discrimination, The American Statistician, 63:4, 366-372, DOI: 10.1198/tast.2009.08210. 